\title{
Wie entsteht psychologische Sicherheit und Teamidentifikation? Eine empirische Untersuchung
}

\author{
Thomas Bachmann • Katherina Quispe Bravo
}

Eingegangen: 4. Mai 2021 / Angenommen: 12. Juni 2021 / Online publiziert: 4. August 2021

(C) Der/die Autor(en) 2021

Zusammenfassung Die Bedeutung von psychologische Sicherheit und Teamidentifikation sowie die Faktoren für deren Entstehung werden im Kontext von New Work und agilem Arbeiten diskutiert. In einer empirischen Studie wurden Teammitglieder nach Merkmalen ihres Teams, der wahrgenommenen psychologischen Sicherheit und der Identifikation mit dem Team befragt. Die Ergebnisse unterscheiden und identifizieren wesentliche Faktoren zur Beschreibung von Arbeitsteam. Weiterhin konnte ein enger Zusammenhang zwischen psychologischer Sicherheit und Teamidentifikation nachgewiesen werden. Unterschiede zwischen beiden Konstrukten liegen auf der Sozialdimension, die als Prädiktor der Teamidentifikation einen Unterschied zur psychologischen Sicherheit markiert.

Schlüsselwörter Teamfaktoren · Psychologische Sicherheit · Teamidentifikation · New Work · Agiles Arbeiten

PD Dr. Thomas Bachmann $(\bowtie)$

Institut für Psychologie, Humboldt-Universität zu Berlin, Unter den Linden 6, 10099 Berlin,

Deutschland

E-Mail: thomas.bachmann@hu-berlin.de

Katherina Quispe Bravo

artop GmbH, Christburger Str. 4, 10405 Berlin, Deutschland

E-Mail: bravo@artop.de 


\title{
How does psychological safety and team identification emerge? An empirical study
}

\begin{abstract}
The importance of psychological safety and team identification as well as the factors for their development are discussed in the context of New Work and agility. In an empirical study, team members were asked about characteristics of their team, perceived psychological safety, and their identification with the team. The results distinguish and identify essential factors to describe work teams. Furthermore, a close relationship between psychological safety and team identification could be demonstrated. Differences between both constructs lie in the social dimension, which as a predictor of team identification marks a difference to psychological safety.
\end{abstract}

Keywords Team characteristics $\cdot$ Psychological safety $\cdot$ Team identification $\cdot$ New Work · Agility

\section{Teamarbeit und Transformation der Arbeitswelt}

Die Arbeits- und Organisationswelt des 21. Jahrhunderts ist von rasanten Entwicklungs- und Transformationsprozessen geprägt, die in enger Wechselwirkung mit den Möglichkeiten und Auswirkungen der Digitalisierung unserer Gesellschaften verbunden sind. Es entstehen neue Formen der Zusammenarbeit (crowd working, agiles Arbeiten etc.), neue Organisationsformen (Holakratie, Soziokratie, evolutionäre Organisation, Purpose Driven Organization etc.), neue Aufgaben- und Rollenanforderungen (Product Owner, Agile Coach etc.), neue spezifische Bezüge zwischen Individuen und Organisationen (remote, virtuell, temporär, global etc.) sowie ein neues Verständnis von Arbeit (New Work), das viele der o.g. Phänomene in sich vereint und darüber hinaus durch den Einsatz bestimmter Methoden und Formate (Kanban, Scrum, Shared Leadership, Empowerment etc.) gekennzeichnet ist (vgl. Bergmann 1997, 2019; Laloux 2014; Oestereich und Schröder 2017; Robertson 2015; Rüther 2017; Schermuly 2016).

Diese Entwicklungen beschleunigen den seit Jahren anhaltenden Trend, dass der überwiegende Teil der Arbeit in Organisationen in Arbeitsgruppen bzw. Teams erledigt wird. Organisationen übernehmen teambasierte Organisationsdesigns, um durch Innovativität, Flexibilität und Kreativität im Wettbewerb bestehen zu können (Bachmann 2019; Mathieu et al. 2019; Scholl 2020). Dabei wird die Teamarbeit zunehmend dynamisiert: Wechselnde Konstellationen von Teammitgliedern, volatile Umwelten, wechselnde komplexe Aufgaben, Mehrfachzugehörigkeiten kennzeichnen u. a. moderne Teamarbeit. Diese erfordert mehr denn je, dass die Teammitglieder intensiv miteinander kommunizieren, gemeinsam Entscheidungen treffen, Probleme lösen, sich verändernde Abhängigkeiten sortieren und Aufgaben erledigen, die sich ebenfalls ständig verändern (Edmondson 2018).

Kozlowski und Ilgen (2006, S. 79) definieren ein Team nach folgenden Merkmalen und grenzen es damit von Gruppen bzw. alltagssprachlichen Teambegriffen $\mathrm{ab}$ : 
- zwei oder mehr Individuen,

- die sozial interagieren (face-to-face oder zunehmend virtuell),

- gemeinsam ein oder mehrere Arbeitsziele teilen,

- zusammengestellt sind, um für die Organisation relevante Aufgaben zu bearbeiten,

- untereinander Abhängigkeiten in Bezug auf Workflow, Ziele und Ergebnisse austarieren,

- unterschiedliche Rollen und Verantwortungen innehaben,

- eingebettet sind in ein organisationales System mit Grenzen und Bezügen zu einem umfassenderen externen Kontext und Aufgabenumfeld.

Am deutlichsten zeigen sich die beschriebenen Entwicklungen auf der methodischen Ebene der Teamarbeit. Hier nehmen die organisationalen, prozessualen, sozialen und psychischen Veränderungen in den täglichen Praktiken Gestalt an.

Agile Methoden haben ihren Ursprung in der Softwareentwicklung (Beck et al. 2001) und unterscheiden sich von traditionellen Projektlogiken vor allem durch Nutzerzentrierung, Flexibilität, Vorläufigkeit und Feedback sowie eine hohe Interaktionsintensität im Team. Heute finden wir agile Teams nicht mehr nur in der Softwareentwicklung, sondern zunehmend auch in Produkt- und Projekt-Teams, losgelöst von Software und -Technologie. Ein agiles Team ist ein komplexes, adaptives, sozio-technisches System, das sowohl aus der Gruppe als einem System als auch aus seinen Individuen und deren Interaktionen als Subsystemen besteht (Whitworth und Biddle 2007). In der Praxis versteht man unter agilem Arbeiten vor allem das Arbeiten mit agilen Methoden und Einstellungen - also die Nutzung von agilen Tools und die Entwicklung eines agilen Mindsets. Agile Tools schließen Methoden wie Design Thinking, Lean Management, Scrum oder Kanban ein. Ein agiles Mindset ist z. B. geprägt von Unterstützungskultur, Lernorientierung, Kundenorientierung, Beweglichkeit, Wissensaustausch, Vertrauen und Kollaboration (Hofert 2018). Agile Teams sind geprägt von einem flexiblen Rollenverständnis und gehen meist Hand in Hand mit Prinzipien geteilter Führung, shared leadership (Moe et al. 2009).

\section{Psychologische Sicherheit}

Agile Teamarbeit - und nicht nur diese - braucht psychologische Sicherheit, also die kollektive Überzeugung der Teammitglieder, dass es sicher ist, im Arbeitsprozess auch interpersonelle Risiken eingehen zu können, d.h. auch abweichende Meinungen $\mathrm{zu}$ vertreten, andere um Unterstützung zu bitten oder ungewöhnliche Ideen $\mathrm{zu}$ verfolgen. In einem psychologisch sicheren Umfeld haben Teammitglieder das Gefühl, dass sie für solche Verhaltensweisen nicht zurückgewiesen, bestraft oder bloßgestellt werden. Sie erkennen die Kompetenzen der anderen an, haben ein intersubjektives Interesse aneinander und verhalten sich einander gegenüber wohlwollend und können sich auf konstruktive Konflikte oder Konfrontationen einlassen. Diese gemeinsame Überzeugung von einer psychologisch sicheren Team-Umgebung führt dazu, dass Mitarbeitende offener kommunizieren, sich trauen, Bedenken zu äußern, und gegenseitig Feedback einholen (Edmondson und Lei 2014). 
Seit der Einführung des Konzepts durch Schein und Bennis (1965), seinem Wiederauftauchen in der Google-Studie von 2012 (Duhigg 2016) und dessen Weiterentwicklung und systematische Untersuchung u.a. durch Edmondson (1999, 2018), hat sich eine beeindruckende und umfangreiche empirische Forschung zu Prädiktoren, Mediatoren, Moderatoren und Outcome-Variablen psychologischer Sicherheit im Organisationskontext entwickelt. Zahlreiche Studien haben die Prädiktoren auf Individual-, Team- und Organisationsebene untersucht, um zu beschreiben, wie unterstützende organisationale Praktiken z. B. Wissensaustausch, Engagement, Kreativität, Innovation und Effektivität durch psychologische Sicherheit befördert werden (Newman et al. 2017).

\subsection{Psychologische Sicherheit in Teams}

Auf Teamebene zählen die Lernorientierung des Teams als Ganzes sowie Arbeitsgestaltung, Führung und die Beziehungsgestaltung unter Teammitgliedern zu den Faktoren, die psychologische Sicherheit entstehen lassen. Die Lernorientierung eines Teams wirkt sich positiv auf die psychologische Sicherheit aus (Edmondson 1999; Frazier et al. 2017; Wong et al. 2010). Ähnlich wie individuelle Lernorientierung beschreibt die Lernorientierung von Teams das Ausmaß, in dem Teams als Ganzes das Vorkommen und Eingestehen von Fehlern als notwendigen Teil ihrer Entwicklung wahrnehmen können. Weiterhin wurden Faktoren der Arbeitsgestaltung - wie die formale Struktur eines Teams (Bresman und Zellmer-Bruhn 2013; Bunderson und Boumgarden 2010), Rollenklarheit (Hackman und Oldham 1976; Tubre und Collins 2000), der Grad der Interdependenz (Wageman 1995) sowie der Grad der Teamautonomie (Turner und Lawrence 1965) - als Bedingungen psychologischer Sicherheit identifiziert (Newman et al. 2017). Aspekte des Kommunikations- und Lernverhaltens - das Ansprechen von Fehlern, Wissensaustausch (Mu und Gnyawali 2003; Siemsen et al. 2009; Xu und Yang 2010; Zhang et al. 2010), Sprechverhalten (Bienefeld und Grote 2014; Detert und Burris 2007; Liang et al. 2012; Tynan 2005), Schweigeverhalten (Brinsfield 2013), zwischenmenschliche Kommunikation - wurden auf Individualebene (Carmeli et al. 2009; Carmeli und Gittell 2009; Liu et al. 2014) wie auch auf Gruppenebene als weitere Effekte gefunden (Bstieler und Hemmert 2010; Carmeli et al. 2009; Edmondson 1999; Ortega et al. 2010; Roberto 2002; Stalmeijer et al. 2007; Van den Bossche et al. 2006; Wong et al. 2010).

Eng verknüpft mit dem Lernverhalten sind Kreativität (z. B. Hirst et al. 2009; Madjar und Ortiz-Walters 2009) und Innovation (z. B. Edmondson 1999), die aus psychologischer Sicherheit resultieren. Die Überzeugung, dass andere Teammitglieder sie nicht für ihre Fehler bestrafen werden, fördert das Vertrauen des Teams in risikofreudige Aktivitäten und das Experimentieren mit noch nicht erprobten Handlungen, was von entscheidender Bedeutung für die Erzeugung von Innovation in Teams ist. West (1990) argumentiert weiter, dass Teams, die ein höheres $\mathrm{Ma}$ an psychologischer Sicherheit durch die Förderung von positivem Teamaffekt und konstruktivem Konfliktmanagement herstellen, kreativer sind (West und Richter 2008). In ähnlicher Weise betonen Kark und Carmeli (2009) die Bedeutung psychologischer Sicherheit in Forschungs- und Entwicklungsteams, in denen Risikobereitschaft und Exploration entscheidend sind; denn explorative Prozesse sind 
naturgemäß mit Gefahren und Unsicherheit verbunden, die zu fehlerhaften Konsequenzen führen können. Positive, tragfähige und vertrauensvolle Beziehungen ermöglichen es, kritische Informationen und Ideen offen zu teilen und auszutauschen, die notwendig sind, um neue Lösungen zu schaffen, neue Arbeitsprozesse zu entwickeln und die Arbeitsleistung zu verbessern (Carmeli et al. 2009; Kahn 1990). Die Entstehung von psychologischer Sicherheit kann durch die Qualität der zwischenmenschlichen Beziehungen erklärt werden: Die Arbeit in einem Team ist mit gegenseitiger Abhängigkeit verbunden, die auf dem Bedürfnis und der Bereitschaft zur Zusammenarbeit basiert, um persönliche und organisatorische Ziele zu erreichen. Aspekte interindividueller Beziehungen - das Ausmaß, in dem Individuen miteinander interagieren (Carmeli et al. 2009; Carmeli und Gittell 2009; May et al. 2004), die Qualität der Beziehungen zwischen den Teammitgliedern (Brueller und Carmeli 2011; Carmeli et al. 2009), die Unterstützung durch Teammitglieder (Frazier et al. 2017; Schepers et al. 2008) und das Vertrauen in Teammitglieder (May et al. 2004; Zhang et al. 2010) - sind Treiber für psychologische Sicherheit im Team.

\subsection{Psychologische Sicherheit und Führung}

Auch das Führungsverhalten hat starken Einfluss auf die wahrgenommene psychologische Sicherheit, da sich Teammitglieder u.a. am Verhalten von Führungspersonen orientieren. Dies betrifft vor allem den Umgang mit Macht. Bis heute wurden zahlreiche Führungskonstrukte als Prädiktoren, zur Strukturierung und Erklärung für die Entstehung von psychologischer Sicherheit untersucht. Positive Führungsstile - z. B. die integrative Führung (Bienefeld und Grote 2014; Carmeli et al. 2010; Hirak et al. 2012; Nembhard und Edmondson 2011), transformationale Führung (Detert und Burris 2007; Nemanich und Vera 2009; Wang und Leung 2011), leader-member exchange (Coombe 2010), ethische Führung (Walumbwa und Schaubroeck 2009), veränderungsorientierte Führung (Ortega et al. 2014), shared leadership (Liu et al. 2014) - wirken sich positiv auf das Lernverhalten von Individuen und die Lernkultur im Team, mediiert durch psychologische Sicherheit, aus (Frazier et al. 2017; Newman et al. 2017).

Die Fülle der Forschungsergebnisse zeigt, dass es sich bei der psychologischen Sicherheit um ein Schlüsselkonzept der Teamarbeit und damit der modernen Arbeitswelt handelt. Zunehmende Unsicherheit, der Wegfall von Strukturen und Grenzen sowie die Zunahme an Selbstführung und Selbstorganisation können nur durch gelingende Kommunikation und Entscheidungsprozesse im Team zu mehr Flexibilität, Kreativität und Innovationen führen. Psychologische Sicherheit kann somit als Basis für gelingende Teamarbeit und Identifikation mit dem Team bzw. Teamidentifikation und somit für agiles Arbeiten und New Work angesehen werden.

\section{Teamidentifikation}

Die Theorie der sozialen Identität (Tajfel und Turner 2004) hat die Forschung zu Intergruppenbeziehungen maßgeblich vorangetrieben. Seit ihrer Entwicklung wurden in zahlreichen empirischen Untersuchungen verschiedene Aspekte der Theorie 
untersucht (Brewer und Kramer 1985; Tajfel 1982). Nach dieser Theorie umfasst das menschliche Selbstkonzept die persönliche Identität „Ich“ und die soziale Identität „Wir“. Dabei beschreibt die persönliche Identität individuelle Fähigkeiten, Vorlieben oder Persönlichkeitseigenschaften, wohingegen sich die soziale Identität aus Attributen sozialer Gruppenzugehörigkeit zusammensetzt (Tajfel und Turner 2004). Tajfel beschreibt die soziale Identität als den Teil des Selbstkonzepts eines Individuums, der sich aus dem Wissen um seine Zugehörigkeit zu einer sozialen Gruppe sowie dem Wert und der emotionalen Bedeutung ergibt, die mit dieser Zugehörigkeit verbunden sind. Aus dieser Definition extrahieren Hinkle et al. (1989) drei sich unterscheidende Aspekte der Identifikation mit einer Gruppe: das Wissen um die Zugehörigkeit, ihre Bewertung und der emotional-affektive Aspekt der Gruppenzugehörigkeit. Van der Vegt und Bunderson (2005) entwickeln den Gedanken der sozialen Identitätstheorie von Tajfel und Turner (2004) weiter und beziehen ihn auf multidisziplinäre Teams in Organisationen. Sie definieren die kollektive Teamidentifikation als die subjektiv wahrgenommene emotionale Verbundenheit des Selbst mit sozialen Arbeitssystem, dem man angehört.

Teamidentifikation wirkt sich positiv auf die Übereinstimmung von individuellen und Teamzielen aus, fördert die intrinsische Motivation (Van Knippenberg und Van Schie 2000), steigert Lernmotivation sowie Lernverhalten der Teammitglieder und verbessert insgesamt die Teamleistung (Van Der Vegt und Bunderson 2005). Außerdem hat die die Teamidentifikation einen positiven Zusammenhang mit Intragruppenkohäsion, Kooperation, Altruismus und einer positiven Wahrnehmung des Teams (Turner 1984). Es ist auch anzunehmen, dass ähnliche Zusammenhänge mit Loyalität sowie Stolz auf das Team und seine Aktivitäten bestehen. Auch Faktoren wie Prestige, Unterscheidbarkeit und eine wahrgenommene Konkurrenz zu anderen Teams können die Teamidentität nachhaltig ausbauen und fördern (Ashforth und Mael 1989). Es ist zu vermuten, dass es in Anlehnung an den Zusammenhang von Statuswahrnehmung und psychologischer Sicherheit einen direkten Zusammenhang zwischen ,in-group“-Zugehörigkeit und Teamidentifikation gibt und damit auch einen Zusammenhang zur psychologischen Sicherheit.

\section{Empirische Studie}

In der folgenden Studie wurde der Zusammenhang zwischen psychologischer Sicherheit und Teamidentifikation untersucht. Aufgrund der bisherigen Befundlage ist davon auszugehen, dass beide Konstrukte in einem engen Zusammenhang stehen; worin sie sich jedoch unterscheiden, soll eine zentrale Fragestellung sein. Weiterhin soll analysiert werden, welche Teamparameter psychologische Sicherheit und Teamidentifikation vorhersagen. Dazu sollen strukturelle und prozessuale Eigenschaften von Teams und von Teamarbeit identifiziert werden, die vor allem in der beschriebenen Transformation zu New Work bedeutsam sind. Dazu gehören z. B. Teameigenschaften wie Hierarchie, wechselnde Zugehörigkeit, Teamgröße, wechselnde Aufgaben, Diversität, Rollenverteilung, Führung etc. Für die Studie wurden Personen, die aktuell Mitglieder eines oder mehrerer Arbeitsteams sind, nach ihrer 
wahrgenommenen psychologischen Sicherheit und ihrer Teamidentifikation sowie nach Eigenschaften ihres Teams befragt.

\subsection{Forschungsfragen}

Da es sich um eine explorative Untersuchung handelt, werden zunächst nur Forschungsfragen auf einer allgemeinen Ebene formuliert:

1. Durch welche strukturellen und prozessualen Parameter lassen sich Arbeitsteams unterscheiden und klassifizieren? Gibt es hier grundlegende strukturelle und prozessuale Faktoren?

2. Welcher Zusammenhang besteht zwischen psychologischer Sicherheit und Teamidentifikation und worin unterscheiden sich beide Konstrukte?

3. Welche strukturellen und prozessualen Faktoren können psychologische Sicherheit und Teamidentifikation prädiktieren?

4. Gibt es unterschiedliche Prädiktoren für psychologische Sicherheit und Teamidentifikation?

\subsection{Erhebungsinstrumente und Untersuchungsdesign}

Bei der Entwicklung der Erhebungsinstrumente standen Kürze und Prägnanz im Vordergrund, um eine möglichst hohe Beteiligung und eine geringe Drop-Out-Quote zu erreichen. Für die Erhebung der psychologischen Sicherheit wurde daher die Kurzskala von Edmondson (1999) ins Deutsche übertragen. Die Items wurden so umformuliert, dass sie einen direkten Bezug zur Team-Ebene herstellen. Zur Teamidentifikation wurde die Skala von Hinkle et al. (1989) verwendet und durch eigene Items ergänzt. Die strukturellen und prozessualen Teameigenschaften wurden mit einem semantischen Differential aus 14 selbst entwickelten Items erfasst. Zusätzlich wurden noch einige sozio-demographische Angaben sowie Daten zur Organisation und zur Dauer der Zugehörigkeit erhoben. Die Skalen mit den dazugehörigen Items sind in Tab. 1 dargestellt.

Die Datenerhebung erfolgte online im Zeitraum von Januar bis Februar 2021. Die Teilnehmenden der Untersuchung wurden aus dem Absolventennetzwerk von artop - Institut an der Humboldt-Universität zu Berlin sowie durch soziale Netzwerke gewonnen. Teilnahmebedingung war, dass die Personen beruflich in einem Team arbeiten. Die Bearbeitungszeit betrug im Median 8 min. 584 Personen klickten auf den Link zum Fragebogen. Davon begannen 301 Personen mit der Bearbeitung des Fragebogens, von denen wiederum 235 den Fragebogen vollständig bearbeiteten. Nach der Datenbereinigung konnten 215 Datensätze ausgewertet werden.

\subsection{Stichprobe}

Es nahmen 143 Frauen und 67 Männer an der Untersuchung teil, eine Person nutzte die Option ,divers“. $85 \%$ der Teilnehmenden waren zwischen 30 und 60 Jahren alt. 168 Personen gaben ein Studium als höchste Qualifikation an. Die Stichprobe umfasst ein breites Branchenspektrum, wobei die IT-Branche mit ca. 20\%, die Dienst- 
Tab. 1 Verwendete Skalen und Items

Psychologische Sicherheit (nach Edmondson 1999)

1. In unserem Team kann man auch Probleme und schwierige Themen offen ansprechen

2. Die Mitglieder unseres Teams sind manchmal Teammitgliedern gegenüber abweisend, die anders sind

3. In unserem Team kann man sich trauen, ein persönliches Risiko einzugehen (z. B. Fehler zugeben oder ungewöhnliche Ideen einbringen)

4. In unserem Team ist es schwierig, andere Teammitglieder um Hilfe zu bitten

5. In unserem Team werden die besonderen Fähigkeiten und Begabungen anderer wertgeschätzt

6. Wenn man in unserem Team einen Fehler macht, dann wird einem das oft vorgehalten

7. Niemand in unserem Team würde absichtlich etwas tun, das meiner Arbeit schadet

Teamidentifikation (nach Hinkle et al. 1989)

1. Ich identifiziere mich mit diesem Team

2. Ich bin froh, diesem Team anzugehören

3. Ich kann in diesem Team richtig aufblühen

4. Ich denke, dieses Team arbeitet gut zusammen

5. Ich sehe mich selbst als einen wichtigen Teil dieses Teams

6. Ich passe gut zu den anderen Mitgliedern dieses Teams

7. Ich halte dieses Team für wichtig

8. Ich fühle mich mit den Mitgliedern dieses Teams wohl

9. Ich fühle mich diesem Team verbunden

10. Ich denke, dieses Team ist erfolgreich

\section{Teameigenschaften}

\section{Eher klein (bis 3 Personen)}

2. Funktioniert überwiegend formell

3. Hierarchisch aufgebaut

$$
\text { 4. Agil arbeitend }
$$

5. Autoritär geführt

6. Stabil und überdauernd

7. Remote arbeitend

8. Organisiert die Arbeit selbst

9. Beziehungsorientiert

(Mitglieder sind wichtig)

10. Feste Teammitglieder

11. Gleichbleibende Aufgaben

12. Verglichen mit anderen sehr erfolgreich

13. Eher homogen

14. Eher innovativ

\begin{tabular}{|c|c|}
\hline- & $\begin{array}{l}\text { Eher groß (mehr } \\
\text { als } 8 \text { Personen) }\end{array}$ \\
\hline- & $\begin{array}{l}\text { Funktioniert über- } \\
\text { wiegend informell }\end{array}$ \\
\hline- & $\begin{array}{l}\text { Heterarchisch (al- } \\
\text { le sind gleich) }\end{array}$ \\
\hline- & Traditionell arbeitend \\
\hline- & Partizipativ geführt \\
\hline- & Flexibel und kurzlebig \\
\hline- & Face-to-face arbeitend \\
\hline- & $\begin{array}{l}\text { Arbeitsabläufe wer- } \\
\text { den vorgegeben }\end{array}$ \\
\hline- & $\begin{array}{c}\text { Aufgabenorientiert } \\
\text { (Ergebnisse sind wichtig) }\end{array}$ \\
\hline- & Wechselnde Teammitglieder \\
\hline- & Wechselnde Aufgaben \\
\hline- & Eher durchschnittlich \\
\hline- & Eher divers \\
\hline- & Eher konventionell \\
\hline
\end{tabular}


leistungsbranche mit $15 \%$, die Gesundheitsbranche mit $8 \%$, der öffentliche Sektor mit $7 \%$, Non-Profit-Organisationen mit $6 \%$ und Wissenschaft und Forschung mit $5 \%$ am stärksten vertreten sind. $25 \%$ der Teilnehmenden arbeiteten im HR-Bereich, $21 \%$ haben interne Beratungsfunktionen, und $10 \%$ arbeiten in der Produktentwicklung. $80 \%$ gaben an, mehr als ein Jahr ihrem Team anzugehören. Damit ist diese Stichprobe hinsichtlich der Zusammensetzung nicht als repräsentativ anzusehen, sondern stellt einen spezifischen Ausschnitt der derzeitigen Arbeitswelt dar.

\subsection{Ergebnisse}

Abb. 1 zeigt die Verteilung der Teamvariablen in der Stichprobe und gibt damit ein Bild über die Eigenschaften der Teams, der die Untersuchungsteilnehmenden angehören. Aufgrund der relativ umfangreichen Stichprobe können die Verteilungen als ein aktuelles Abbild von Merkmalen von Arbeitsteams in den vorkommenden Branchen betrachtet werden. Hervorzuheben ist, dass die Teilnehmenden ihre Teams überwiegend als informell, heterarchisch, agil, partizipativ, stabil, remote arbeitend, selbstorganisiert, beziehungsorientiert, mit festen Mitgliedern, erfolgreich und innovativ beschreiben. Der große Anteil an remote arbeitenden Teams ist durch die Bedingungen in der Corona-Pandemie zu erklären.

\section{Semantisches Differential der Teamvariablen}

Prozentuale Antwortverteilung $(N=215)$

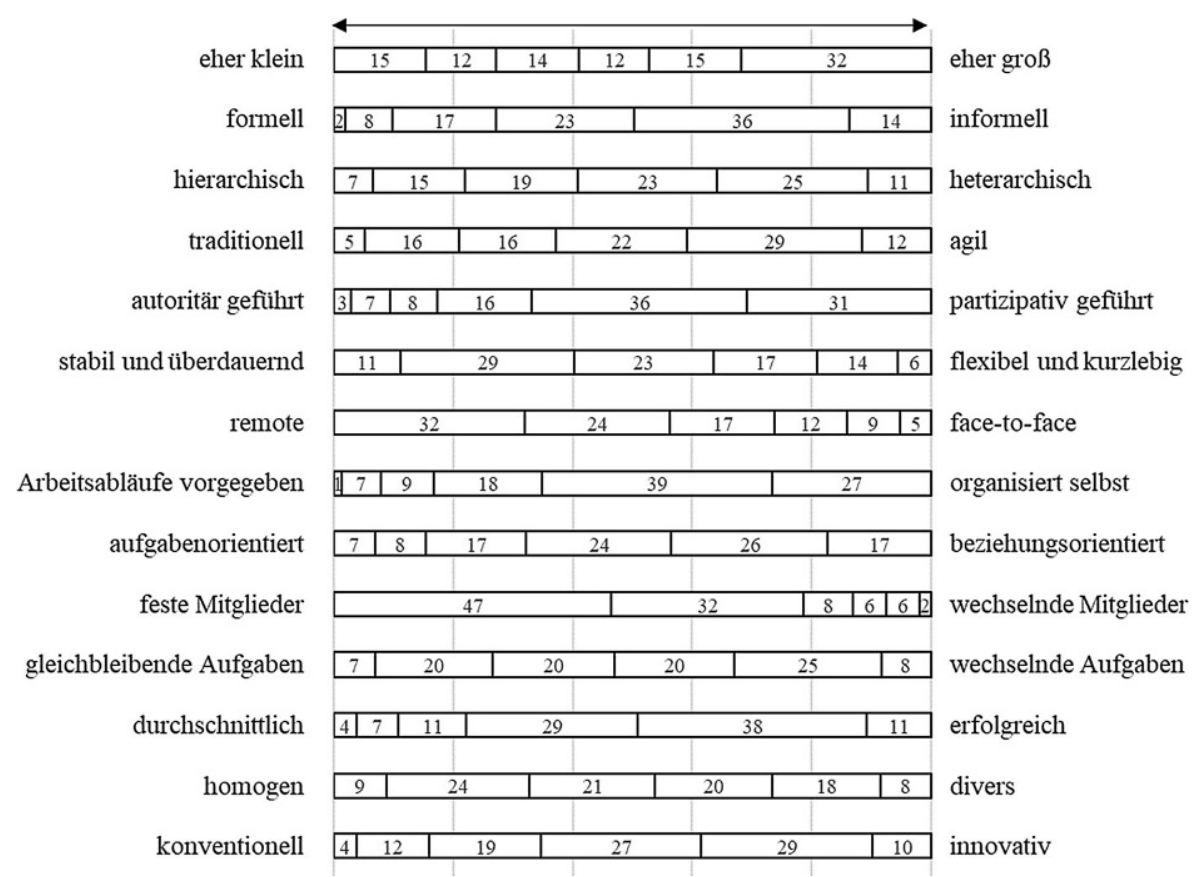

Abb. 1 Prozentuale Antwortverteilung der Teamvariablen im semantischen Differential (bipolare 6-stufige Skala ohne Skalenpunktbenennungen) 
Tab. 2 Faktorenstruktur der Teameigenschaften (Varimax-rotierte Komponentenmatrix mit Ladungen $>0,20$ )

\begin{tabular}{|c|c|c|c|c|c|}
\hline \multirow[t]{2}{*}{ Faktoren } & \multicolumn{5}{|c|}{ Faktorladungen } \\
\hline & 1 & 2 & 3 & 4 & 5 \\
\hline \multicolumn{6}{|l|}{ Faktor 1: Selbstorganisation } \\
\hline Eher innovativ - eher konventionell & $\mathbf{0 , 7 8}$ & - & - & $-0,22$ & - \\
\hline Agil arbeitend - traditionell arbeitend & $\mathbf{0 , 7 7}$ & - & - & - & - \\
\hline Autoritär geführt - partizipativ geführt & $-0,74$ & - & - & - & - \\
\hline $\begin{array}{l}\text { Hierarchisch aufgebaut - heterarchisch (alle } \\
\text { sind gleich) }\end{array}$ & $-0,68$ & - & - & - & - \\
\hline $\begin{array}{l}\text { Organisiert die Arbeit selbst - Arbeitsabläufe } \\
\text { werden vorgegeben }\end{array}$ & 0,66 & - & 0,43 & - & - \\
\hline $\begin{array}{l}\text { Verglichen mit anderen sehr erfolgreich - } \\
\text { eher durchschnittlich }\end{array}$ & 0,63 & 0,27 & $-0,43$ & - & - \\
\hline \multicolumn{6}{|l|}{ Faktor 2: Flexibilität } \\
\hline $\begin{array}{l}\text { Stabil und überdauernd - flexibel und kurzle- } \\
\text { big }\end{array}$ & $-0,21$ & 0,77 & - & - & - \\
\hline $\begin{array}{l}\text { Feste Teammitglieder - wechselnde Teammit- } \\
\text { glieder }\end{array}$ & - & 0,76 & - & - & - \\
\hline $\begin{array}{l}\text { Gleichbleibende Aufgaben - wechselnde } \\
\text { Aufgaben }\end{array}$ & - & 0,50 & - & 0,48 & $-0,22$ \\
\hline \multicolumn{6}{|l|}{ Faktor 3: Teamgröße } \\
\hline $\begin{array}{l}\text { Eher klein (bis } 3 \text { Personen) - eher groß (mehr } \\
\text { als } 8 \text { Personen) }\end{array}$ & - & - & 0,86 & - & - \\
\hline \multicolumn{6}{|l|}{ Faktor 4: Diversität } \\
\hline Eher homogen - eher divers & - & - & - & $\mathbf{0 , 9 0}$ & - \\
\hline \multicolumn{6}{|l|}{ Faktor 5: Intersubjektivität } \\
\hline Remote arbeitend - face-to-face arbeitend & 0,48 & - & - & - & 0,66 \\
\hline $\begin{array}{l}\text { Funktioniert überwiegend formell - funktio- } \\
\text { niert überwiegend informell }\end{array}$ & $-0,40$ & 0,33 & - & - & $\mathbf{0 , 5 9}$ \\
\hline $\begin{array}{l}\text { Beziehungsorient. (Mitglieder wichtig) - } \\
\text { aufgabenorient. (Ergebnisse wichtig) }\end{array}$ & 0,41 & 0,28 & - & - & $-\mathbf{0 , 5 1}$ \\
\hline
\end{tabular}

Im nächsten Schritt wurden die verwendeten Skalen hinsichtlich ihrer Struktur und internen Konsistenz untersucht. Faktorenanalysen (Hauptkomponentenanalyse) der Skalen für die psychologische Sicherheit und der Teamidentifikation ergaben keine Hinweise auf Unterfaktoren in den Skalen (Kriterien: Eigenwert >1, ScreeTest). Die interne Konsistenz (Cronbachs $\alpha$ ) betrug $\alpha=0,82$ für die Skala psychologische Sicherheit und $\alpha=0,95$ für die Skala Teamidentifikation. Damit können beiden Skalen sehr gute Messeigenschaften bescheinigt werden. Allerdings differenziert die Skala Teamidentifikation nicht nach den Komponenten Wissen, Bewertung und emotional-affektive Identifikation, wie von Hinkle et al. (1989) intendiert, da sie nur aus einem Faktor besteht (Eigenwert=6,94).

Die Faktorenanalyse für die Teameigenschaften ergab ein interessantes Ergebnis. Danach liegt bei diesem Itemblock eine 5-Faktoren-Struktur vor, die in Tab. 2 dargestellt ist. Die Faktorenanalyse zeigt, dass die Teilnehmenden ihre Teams auf fünf grundlegenden Dimensionen wahrnehmen, die in der Benennung der Faktoren 
(Selbstorganisation, Flexibilität, Teamgröße, Diversität und Intersubjektivität) zum Ausdruck kommen. Die Tabelle der Eigenwerte (Tab. 3) zeigt, dass vor allem der fünfte Faktor nur noch unbedeutend zur Varianzaufklärung beiträgt und hohe Nebenladungen auf den ersten Faktor hat. Die interne Konsistenzanalyse ergibt dementsprechend nur für den Faktor ,Selbstorganisation“ eine gute Reliabilität von $\alpha=0,82$. Die anderen beiden Faktoren, „Flexibilität“ und „Intersubjektivität“, ergeben leider keine konsistenten Skalen. Aus diesem Grund gingen die Teameigenschaften in die folgenden Analysen als Einzelitems ein. Das Ergebnis der Faktorenanalyse kann weiterhin als ein Beleg für die Konstruktvalidität des semantischen Differentials angesehen werden, da sich inhaltlich ähnliche Dimensionen auf gemeinsame Faktoren vereinen. Damit kann davon ausgegangen werden, dass die Befragten die Items des Fragebogens mit hoher inhaltlicher Übereinstimmung verstanden haben. Dies trifft, wie im Folgenden deutlich wird, auch auf die Skalen für psychologische Sicherheit und Teamidentifikation zu.

Im nächsten Schritt wurden der Zusammenhang zwischen psychologischer Sicherheit und Teamidentifikation sowie die Prädiktion beider Konstrukte durch die Teameigenschaften analysiert. Eine Korrelationsanalyse ergab einen Zusammenhang zwischen psychologischer Sicherheit und Teamidentifikation von $r=0,76(p=0,000)$

Tab. 3 Erklärte Varianz der Faktoren der Teameigenschaften

\begin{tabular}{llll}
\hline Faktor & \multicolumn{2}{l}{ Anfängliche Eigenwerte } \\
& Gesamt & $\%$ der Varianz & Kumulierte \% \\
\hline Selbstorganisation & 3,84 & 27,41 & 27,41 \\
Stabilität & 1,76 & 12,55 & 39,96 \\
Teamgröße & 1,25 & 8,91 & 48,86 \\
Diversität & 1,10 & 7,86 & 56,73 \\
Intersubjektivität & 1,02 & 7,31 & 64,04 \\
\hline
\end{tabular}

Tab. 4 Korrelationen der Teameigenschaften mit psychologischer Sicherheit und Teamidentifikation

\begin{tabular}{lll}
\hline Teamvariablen & Psychologische Sicherheit & Teamidentifikation \\
\hline Eher groß (mehr als 8 Personen) & $-0,07$ & 0,02 \\
Funktioniert überwiegend informell & $0,33^{* *}$ & $0,24^{* *}$ \\
Heterarchisch (alle sind gleich) & $0,40^{* *}$ & $0,31^{* *}$ \\
Agil arbeitend & $0,43^{* *}$ & $0,43^{* *}$ \\
Partizipativ geführt & $0,58^{* *}$ & $0,51^{* *}$ \\
Flexibel und kurzlebig & 0,06 & 0,04 \\
Face-to-face arbeitend & $\mathbf{- 0 , 2 2}^{* *}$ & $-0,08$ \\
Organisiert die Arbeit selbst & $0,38^{* *}$ & $0,30^{* *}$ \\
Beziehungsorientiert (Mitglieder wichtig) & $0,33^{* *}$ & $0,43^{* *}$ \\
Wechselnde Teammitglieder & $-0,12$ & $-\mathbf{0 , 1 9 * *}$ \\
Wechselnde Aufgaben & 0,10 & 0,13 \\
Verglichen mit anderen sehr erfolgreich & $0,41^{* *}$ & $0,47^{* *}$ \\
Eher divers & 0,04 & 0,04 \\
Eher innovativ & $0,50^{* *}$ & $0,52^{* *}$ \\
\hline
\end{tabular}
*** $p<0,01$ 
Tab. 5 Multiple Regressionsanalyse zur Vorhersage von psychologischer Sicherheit im Team

\begin{tabular}{llll}
\hline Team-Variablen & $\beta$ & $t$ & $p$ \\
\hline Funktioniert überwiegend informell & 0,15 & 2,63 & 0,009 \\
Partizipativ geführt & 0,39 & 6,24 & 0,000 \\
Verglichen mit anderen sehr erfolgreich & 0,16 & 2,65 & 0,009 \\
Eher innovativ & 0,20 & 2,95 & 0,004 \\
\hline
\end{tabular}

$R^{2}=0,44 ; F(4)=39,07 ; p=0,000$

und damit eine gemeinsame Varianz von $58 \%$. Beide Konstrukte sind - wie erwartet - eng verwandt. Für die folgenden Analysen werden daher die Unterschiede im Fokus stehen. Zunächst wurden die Produkt-Moment-Korrelationen zwischen den Teameigenschaften und psychologischer Sicherheit und Teamidentifikation berechnet. Diese zeigen für beide Konstrukte ein ähnliches Muster, abgesehen von zwei Ausnahmen: Bei der psychologischen Sicherheit gibt es eine signifikante negative Korrelation mit der Teameigenschaft ,remote vs. face-to-face arbeitend“ und bei der Teamidentifikation eine signifikante negative Korrelation mit der Teameigenschaft „feste vs. wechselnde Teammitglieder“. Die Teamgröße, Teamdauer, wechselnde Aufgaben und Diversität zeigen keinen Zusammenhang mit psychologischer Sicherheit und Teamidentifikation. Systematische Zusammenhänge zu beiden Konstrukten gibt es mit den Teamvariablen informell, heterarchisch, agil arbeitend, partizipativ, selbstorganisiert, beziehungsorientiert, erfolgreich und innovativ (Tab. 4).

Die korrelativen Zusammenhänge werden in den folgenden multiplen BackwardRegressionsanalysen genauer betrachtet. Dieses Verfahren bietet gegenüber den Korrelationsanalysen den Vorteil, dass redundante Prädiktoren (hohe Interkorrelationen zwischen Prädiktoren) sowie Suppressionseffekte (verschiedene Varianzanteile der Variablen werden in der Korrelationsanalyse nicht berücksichtigt) analysiert werden und somit nur Variablen schrittweise in der Regressionsgleichung verbleiben, die einen von anderen Prädiktoren unabhängigen Zusammenhang zur Zielgröße herstellen können (vgl. Bortz 2013). In der ersten Regressionsanalyse wurden alle 14 Variablen der Teameigenschaften als Prädiktoren für die psychologische Sicherheit untersucht. Dabei zeigten sich nach dem Backward-Verfahren vier Variablen als signifikante Prädiktoren (Tab. 5), die hier nur mit einem Pol in gleichsinniger Merkmalsrichtung dargestellt sind.

Im Ergebnis der Regressionsanalyse zeigt sich, dass die Arbeit im Team von den Teammitgliedern dann als psychologisch sicher wahrgenommen wird, wenn die Führungsaufgabe partizipativ erledigt wird, das Team als innovativ und erfolgreich wahrgenommen wird sowie durch informelle Kommunikation geprägt ist.

Die Teamidentifikation hingegen wird durch sechs Variablen prädiktiert (Tab. 6). Neben der partizipativen Führung, der Innovativität und dem Erfolg des Teams erweisen sich die Agilität, die Beziehungsorientierung sowie face-to-face-Arbeit (also in Präsenz und nicht remote) als weitere Prädiktoren. Das Ergebnis zeigt, das sich die Teamidentifikation von der psychologischen Sicherheit vor allem durch eine starke Gewichtung der persönlichen Nähe und Beziehungen unterscheidet. Interessant ist weiterhin, dass sich die Variablen wie Teamgröße, Dauer der Zugehörigkeit, wech- 
Tab. 6 Multiple Regressionsanalyse zur Vorhersage von Teamidentifikation

\begin{tabular}{llll}
\hline Teamvariablen & $\beta$ & $t$ & $p$ \\
\hline Agil arbeitend & 0,12 & 1,68 & 0,095 \\
Partizipativ geführt & 0,24 & 3,79 & 0,000 \\
Face-to-face arbeitend & 0,10 & 1,79 & 0,075 \\
Beziehungsorientiert & 0,24 & 4,27 & 0,000 \\
Verglichen mit anderen sehr erfolgreich & 0,20 & 3,16 & 0,002 \\
Eher innovativ & 0,19 & 2,65 & 0,009 \\
\hline
\end{tabular}

$R^{2}=0,47 ; F(6)=28,91 ; p=0,000$

selnde Mitglieder oder Aufgaben sowie Diversität des Teams in diesen Analysen nicht als signifikante Prädiktoren erweisen.

Zur Verdeutlichung der Wirkung der Teamfaktoren wurden in einer weiteren Analyse die Fälle bezüglich der psychologischen Sicherheit in Quartile aufgeteilt und anschließend das obere und das untere Quartil einem Mittelwertvergleich unterzogen. Dabei ergab sich für das untere Quartil (Q1) eine mittlere psychologische Sicherheit von $M=3,86(S D=0,53 ; N=54)$ und für das obere Quartil (Q4) von $M=5,81(S D=0,15 ; N=53)$. Die geringe Streuung im oberen Quartil weist auf eine rechtschiefe Verteilung der Variablen hin, deren Mittelwert in der gesamten Stichprobe bei $M=4,95(S D=0,79 ; N=215)$ liegt. Die Unterschiede zwischen den beiden Quartilgruppen hinsichtlich der Mittelwertunterschiede sind in Tab. 7 dargestellt. Die Ergebnisse vertiefen die Ergebnisse aus den Regressionsanalysen und zeigen noch deutlicher, dass die Teamstruktur-Merkmale wie Größe, Dauer, wechselnde Mitglieder und Aufgaben oder Diversität nicht mit der psychologischen Sicherheit in Zusammenhang stehen. Nimmt man die Teamfaktoren, die im Ergebnis der Faktorenanalyse entstanden sind, zeigt sich, dass als Prädiktoren für die wahrgenommene psychologische Sicherheit der Grad an Selbstorganisation und Intersubjektivität relevant sind.

\section{Zusammenfassung und Ausblick}

In der vorliegenden Studien wurden Teammitglieder nach charakteristischen Teammerkmalen sowie nach der wahrgenommenen psychologischen Sicherheit und der Teamidentifikation befragt. Ein erstes interessantes Ergebnis entstand durch die explorative Faktorenanalyse der Teamvariablen. Hier konnten zwei Faktorengruppen mit insgesamt fünf Faktoren identifiziert werden:

1. Strukturelle Merkmale: Größe, Flexibilität und Diversität

2. Prozessuale Merkmale: Selbstorganisation und Intersubjektivität

Die weiterführenden Analysen zeigten, dass ausschließlich die prozessualen Teammerkmale Zusammenhänge zur psychologischen Sicherheit und Teamidentifikation aufweisen. Dies bedeutet im Umkehrschluss, dass herausfordernde strukturelle Gegebenheiten der Teamarbeit in modernen Organisationen wie wechselnde Aufgaben und Mitglieder, Diversität oder die Teamgröße durch gute Prozessge- 
Tab. 7 Ergebnisse der Varianzanalyse für den Quartilvergleich (oberes und unteres Quartil der Stichprobe bzgl. psychologischer Sicherheit; Q1: $N=54$; Q4: $N=53$; DF 1, 105)

\begin{tabular}{|c|c|c|c|c|}
\hline Teamvariablen & $\begin{array}{l}M \text { unteres Quar- } \\
\text { til }\end{array}$ & $\begin{array}{l}M \text { oberes Quar- } \\
\text { til }\end{array}$ & $F$ & $p$ \\
\hline Eher klein - eher groß & 4,02 & 3,91 & 0,10 & 0,775 \\
\hline Formell - informell & 3,52 & 4,63 & 23,26 & 0,000 \\
\hline Hierarchisch - heterarchisch & 2,85 & 4,25 & 26,91 & 0,000 \\
\hline Traditionell - agil & 2,94 & 4,58 & 42,53 & 0,000 \\
\hline Autoritär geführt - partizipativ geführt & 3,52 & 5,45 & 79,23 & 0,000 \\
\hline $\begin{array}{l}\text { Stabil und überdauernd - flexibel und } \\
\text { kurzlebig }\end{array}$ & 3,02 & 3,06 & 0,02 & 0,892 \\
\hline Remote - face-to-face & 3,09 & 2,13 & 12,42 & 0,001 \\
\hline $\begin{array}{l}\text { Arbeitsabläufe vorgegeben - organisiert } \\
\text { selbst }\end{array}$ & 4,02 & 5,19 & 25,12 & 0,000 \\
\hline $\begin{array}{l}\text { Aufgabenorientiert - beziehungsorien- } \\
\text { tiert }\end{array}$ & 3,35 & 4,48 & 15,58 & 0,000 \\
\hline $\begin{array}{l}\text { Feste Mitglieder - wechselnde Mitglie- } \\
\text { der }\end{array}$ & 2,15 & 1,91 & 0,91 & 0,345 \\
\hline $\begin{array}{l}\text { Gleichbleibende Aufgaben - wechseln- } \\
\text { de Aufgaben }\end{array}$ & 3,56 & 3,85 & 1,15 & 0,286 \\
\hline Durchschnittlich - erfolgreich & 3,64 & 4,83 & 28,86 & 0,000 \\
\hline Homogen - divers & 3,46 & 3,43 & 0,01 & 0,929 \\
\hline Konventionell - innovativ & 2,91 & 4,53 & 51,02 & 0,000 \\
\hline
\end{tabular}

staltung kompensiert werden können. Es geht also um die Förderung von positiver Interaktionsqualität und Teamkultur unter den jeweils gegebenen organisationalen Bedingungen.

Für die beiden Skalen „psychologische Sicherheit“ und „Teamidentifikation“ konnten durch die Analysen sehr gute Messeigenschaften ermittelt werden. Die multiplen Regressionsanalysen zeigten anschließend, dass die Prädiktoren für die psychologische Sicherheit partizipative Führung, informelle Beziehungen, Innovativität und Teamerfolg sind. Es ist anzunehmen, dass Innovativität und Teamerfolg mit Stimmungen und Wahrnehmungen der Teammitglieder konfundiert sind, die sich bei ausbleibendem Erfolg oder nachlassender Innovativität negativ auf das soziale Miteinander auswirken können und damit auch die wahrgenommene psychologische Sicherheit mindern. Damit bleiben die Partizipation und das informelle Miteinander die wichtigsten Prädiktoren. Dieses Ergebnis ist damit konsistent zu den Studien z. B. von Carmeli et al. (2009), Kahn (1990), Newman et al. (2017) oder Turner und Lawrence (1965), die die Qualität der interindividuellen Beziehungen und den Grad an Teamautonomie als Prädiktoren für Vertrauen bzw. psychologische Sicherheit untersucht haben.

Bei der Prädiktion der Teamidentifikation treten neben Innovativität, Erfolg und Partizipation jetzt auch die Beziehungsorientierung sowie Agilität und face-to-faceInteraktion als Prädiktoren in Erscheinung. Trotz der hohen korrelativen Übereinstimmung von psychologischer Sicherheit und Teamidentifikation zeigt sich hier deutlich, worin sich beide Konstrukte unterscheiden: Innovativität, Mitbestimmung und Erfolg verleihen Sicherheit in der Interaktion mit anderen, Identifikation je- 
doch entsteht erst durch intensive beziehungsorientierte Interaktion in körperlicher Präsenz. Dieses Ergebnis verweist in seiner Eindeutigkeit auf die großen Themen der Arbeits- und Organisationsgestaltung, wie sie im Verlauf der Corona-Pandemie zutage treten. Homeoffice, anstrengende, entfremdete, kanalreduzierte, mikro-asynchrone Kommunikation über Videoplattformen, fehlende persönliche Treffen und Abstimmungsmöglichkeiten, Entgrenzungserscheinungen zwischen Arbeit und Privatleben, Zoom-Fatigue etc. schlagen sich vor allem auf der Dimension der sozialen Zugehörigkeit des Gruppenraums (Yalom und Leszcz 2020) nieder und führen dazu, dass sich Teammitglieder weniger mit ihrem Team identifizieren. Welche Auswirkungen fehlende Identifikation auf die gemeinsame Zielorientierung sowie auf Engagement und Motivation haben, zeigen die Untersuchungen von Van Knippenberg und Van Schie (2000) sowie von Van Der Vegt und Bunderson (2005).

Ein interessantes Zusatzergebnis ist, dass die remote-Interaktion positiv mit der psychologischen Sicherheit korreliert. Dieses Ergebnis kann nur dahingehend gedeutet werden, dass sich Teammitglieder, die nur über Bildschirme miteinander agieren, persönlich sicherer vor Kritik, Ablehnung, Angriffen, Bloßstellungen, abfälligen Kommentaren etc. fühlen. Dies lässt sich aus den Erfahrungen mit Videokonferenzen erklären: Die Interaktion verläuft langsamer, teilweise schleppend, viele Teilnehmende sind passiv, nur ein Bruchteil der Mitteilungen, die üblicherweise in Präsenzmeetings gesendet werden, entstehen im virtuellen Raum bzw. werden dort wahrgenommen. Nur die wichtigsten Dinge werden gesagt oder eingebracht. Alle überlegen, ob ihre Beiträge wichtig sind, und es ist zudem nicht so leicht zu Wort zu kommen, einfach Dazwischenreden oder mit dem Nachbarn tuscheln ist schwierig. Alle wollen nur noch fertig werden und das Treffen nicht länger als nötig ausweiten. Einen interessanten Beitrag dazu verfasste Murphy (2020), in dem diese Phänomene anschaulich dargestellt sind.

\section{Limitationen und Ausblick}

Die vorliegende Studie und deren Ergebnisse werfen weitere Fragen auf. Zunächst sollten die Teamvariablen durch weitere Dimensionen ergänzt und daraus ein solides Instrumentarium zur Charakteristik von Arbeitsteams entwickelt werden, das als Grundlage für weitere Untersuchungen dienen kann. Weiterführende Studien sollten hinsichtlich der Stichprobe auf Repräsentativität bedacht sein sowie andere objektive Kriterien, z. B. Erfolgsmaße der Teamarbeit, mit einbeziehen. Auch sollte die Ergebnisinterpretation unter den Auswirkungen der Corona-Krise und des Lockdowns zur Zeit der Datenerhebung relativiert werden. Hier ist ein Einfluss auf die Wahrnehmung und das Antwortverhalten anzunehmen, zumal etwa ein Drittel der Befragten angab, das ihr Team überwiegend remote arbeitet. Andererseits gaben nur $17 \%$ der Befragten an, weniger als ein Jahr zu ihrem Team zu gehören. Die Antworten speisen sich also überwiegend aus einem längeren Erfahrungszeitraum der Teamarbeit, was den Einfluss der Corona-Situation auf die Daten wiederum relativiert. 
Funding Open Access funding enabled and organized by Projekt DEAL.

Open Access Dieser Artikel wird unter der Creative Commons Namensnennung 4.0 International Lizenz veröffentlicht, welche die Nutzung, Vervielfältigung, Bearbeitung, Verbreitung und Wiedergabe in jeglichem Medium und Format erlaubt, sofern Sie den/die ursprünglichen Autor(en) und die Quelle ordnungsgemäß nennen, einen Link zur Creative Commons Lizenz beifügen und angeben, ob Änderungen vorgenommen wurden.

Die in diesem Artikel enthaltenen Bilder und sonstiges Drittmaterial unterliegen ebenfalls der genannten Creative Commons Lizenz, sofern sich aus der Abbildungslegende nichts anderes ergibt. Sofern das betreffende Material nicht unter der genannten Creative Commons Lizenz steht und die betreffende Handlung nicht nach gesetzlichen Vorschriften erlaubt ist, ist für die oben aufgeführten Weiterverwendungen des Materials die Einwilligung des jeweiligen Rechteinhabers einzuholen.

Weitere Details zur Lizenz entnehmen Sie bitte der Lizenzinformation auf http://creativecommons.org/ licenses/by/4.0/deed.de.

\section{Literatur}

Ashforth, B.E., \& Mael, F. (1989). Social identity theory and the organization. Academy of management review, 14(1), 20-39.

Bachmann, T. (2019). Gruppenintelligenz - gemeinsam sind wir besser? Gruppe Interaktion Organisation, 50, 397-411. https://doi.org/10.1007/s11612-019-00490-0.

Beck, et al. (2001). Manifesto for agile software development. http://agilemanifesto.org. Zugegriffen: 30. Apr. 2021.

Bergmann, F. (1997). Die neue Arbeit: Skizze mit Vorschlag. Gewerkschaftliche Monatshefte, 48(9/10), 524-534.

Bergmann, F. (2019). New work new culture: Work we want and a culture that strengthens us. Hampshire: John Hunt Publishing.

Bienefeld, N., \& Grote, G. (2014). Speaking up in ad hoc multiteam systems: Individual-level effects of psychological safety, status, and leadership within and across teams. European journal of work and organizational psychology, 23(6), 930-945.

Bortz, J. (2013). Statistik: Für Sozialwissenschaftler. Berlin: Springer.

Van den Bossche, P., Gijselaers, W.H., Segers, M., \& Kirschner, P. A. (2006). Social and cognitive factors driving teamwork in collaborative learning environments: team learning beliefs and behaviors. Small Group Research, 37, 490-521.

Bresman, H., \& Zellmer-Bruhn, M. (2013). The structural context of team learning: effects of organizational and team structure on internal and external learning. Organization Science, 24, 1120-1139.

Brewer, M.B., \& Kramer, R.M. (1985). The psychology of intergroup attitudes and behavior. Annual review of psychology, 36(1), 219-243.

Brinsfield, T.C. (2013). Employee silence motives: investigation of dimensionality and development of measures. Journal of Organizational Behavior, 34, 671-697.

Brueller, D., \& Carmeli, A. (2011). Linking capacities of high-quality relationships to team learning and performance in service organizations. Human Resource Management, 50, 455-477.

Bstieler, L., \& Hemmert, M. (2010). Increasing learning and time efficiency in interorganizational new product development teams. Journal of Product Innovation Management, 27, 485-499.

Bunderson, J.S., \& Boumgarden, P. (2010). Structure and learning in self-managed teams: why "bureaucratic" teams can be better learners. Organization Science, 21, 609-624.

Carmeli, A., \& Gittell, J.H. (2009). High-quality relationships, psychological safety, and learning from failures in work organizations. Journal of Organizational Behavior, 30, 709-729.

Carmeli, A., Brueller, D., \& Dutton, J.E. (2009). Learning behaviours in the workplace: the role of high-quality interpersonal relationships and psychological safety. Systems Research and Behavioral Science, 26, 81-98.

Carmeli, A., Reiter-Palmon, R., \& Ziv, E. (2010). Inclusive leadership and employee involvement in creative tasks in the workplace: the mediating role of psychological safety. Creativity Research Journal, $22,250-260$. 
Coombe, D. D. (2010). Secure base leadership: a positive theory of leadership incorporating safety, exploration, and positive action. Doctoral dissertation, Case Western Reserve University.

Detert, J.R., \& Burris, E.R. (2007). Leadership behavior and employee voice: Is the door really open? Academy of Management Journal, 50, 869-884.

Duhigg, C. (2016). What Google learned from its quest to build the perfect team. The New York Times Magazine, 26(2016).

Edmondson, A.C. (1999). Psychological safety and learning behavior in work teams. Administrative science quarterly, 44(2), 350-383.

Edmondson, A.C. (2018). The fearless organization: creating psychological safety in the workplace for learning, innovation, and growth. Hoboken: John Wiley \& Sons.

Edmondson, A. C., \& Lei, Z. (2014). Psychological safety: The history, renaissance, and future of an interpersonal construct. Annu. Rev. Organ. Psychol. Organ. Behav., 1(1), $23-43$.

Frazier, M.L., Fainshmidt, S., Klinger, R. L., Pezeshkan, A., \& Vracheva, V. (2017). Psychological safety: a meta-analytic review and extension. Personnel Psychology, 70(1), 113-165.

Hackman, J. R., \& Oldham, G. R. (1976). Motivation through the design of work: test of a theory. Organizational Behavior and Human Performance, 16, 250-279.

Hinkle, S., Taylor, L. A., Fox-Cardamone, D. L., \& Crook, K. F. (1989). Intragroup identification and intergroup differentiation: a multicomponent approach. British Journal of Social Psychology, 28(4), 305-317.

Hirak, R., Pang, A.C., Carmeli, A., \& Schaubroeck, J. M. (2012). Linking leader inclusiveness to work unit performance: the importance of psychological safety and learning from failures. The Leadership Quarterly, 23, 107-117.

Hirst, G., Van Knippenberg, D., \& Zhou, J. (2009). A cross-level perspective on employee creativity: goal orientation, team learning behavior, and individual creativity. Academy of management journal, 52(2), 280-293.

Hofert, S. (2018). Das agile Mindset. Wiesbaden: Springer.

Kahn, W. A. (1990). Psychological conditions of personal engagement and disengagement at work. Academy of management journal, 33(4), 692-724.

Kark, R., \& Carmeli, A. (2009). Alive and creating: the mediating role of vitality and aliveness in the relationship between psychological safety and creative work involvement. Journal of Organizational Behavior, 30, 785-804.

Van Knippenberg, D., \& Van Schie, E. C. (2000). Foci and correlates of organizational identification. Journal of occupational and organizational psychology, 73(2), 137-147.

Kozlowski, S. W., \& Ilgen, D. R. (2006). Enhancing the effectiveness of work groups and teams. Psychological Science in the Public Interest, 7(3), 77-124.

Laloux, F. (2014). Reinventing organizations: a guide to creating organizations inspired by the next stage in human consciousness. : Nelson Parker.

Liang, J., Farh, C., \& Farh, J. (2012). Psychological antecedents of promotive and prohibitive voice: a twowave examination. Academy of Management Journal, 55, 71-92.

Liu, S., Hu, J., Li, Y., Wang, Z., \& Lin, X. (2014). Examining the cross-level relationship between shared leadership and learning in teams: evidence from China. The Leadership Quarterly, 25, 282-295.

Madjar, N., \& Ortiz-Walters, R. (2009). Trust in supervisors and trust in customers: their independent, relative, and joint effects on employee performance and creativity. Human Performance, 22, 128-142.

Mathieu, J. E., Gallagher, P. T., Domingo, M. A., \& Klock, E. A. (2019). Embracing complexity: reviewing the past decade of team effectiveness research. Annual Review of Organizational Psychology and Organizational Behavior, 6, 17-46.

May, D. R., Gilson, R.L., \& Harter, L.M. (2004). The psychological conditions of meaningfulness, safety and availability and the engagement of the human spirit at work. Journal of occupational and organizational psychology, 77(1), 11-37.

Moe, N.B., Dingsyr, T., \& Kvangardsnes, O. (2009). Understanding shared leadership in agile development: a case study. In 2009 42nd Hawaii international conference on system sciences. IEEE. (S. 1-10).

Mu, S.H., \& Gnyawali, D.R. (2003). Developing synergistic knowledge in student groups. Journal of Higher Education, 74, 689-711.

Murphy, K. (2020). Why zoom is terrible. The New York Times, 23.

Nemanich, L. A., \& Vera, D. (2009). Transformational leadership and ambidexterity in the context of an acquisition. The Leadership Quarterly, 20, 19-33. 
Nembhard, I. M., \& Edmondson, A.C. (2011). Psychological safety: a foundation for speaking up, collaboration, and experimentation. In K. S. Cameron \& G. M. Spreitzer (Hrsg.), The Oxford handbook of positive organizational scholarship. Oxford: Oxford University Press.

Newman, A., Donohue, R., \& Nathan, E. (2017). Psychological safety: a systematic review of the literature. Human Ressource Management Review, 27(3), 521-535.

Oestereich, B., \& Schröder, C. (2017). Das kollegial geführte Unternehmen: Ideen und Praktiken für die agile Organisation von morgen. München: Vahlen.

Ortega, A., Van den Bossche, P., Sanchez-Manzanares, M., Rico, R., \& Gil, F. (2014). The influence of change-oriented leadership and psychological safety on team learning in healthcare teams. Journal of Business and Psychology, 29, 311-321.

Ortega, A., Sanchez-Manzanares, M., Gil, F., \& Rico, R. (2010). Team learning and effectiveness in virtual project teams: the role of beliefs about interpersonal context. Spanish Journal of Psychology, 13, 267-276.

Roberto, M. A. (2002). Lessons from everest: the interaction of cognitive bias, psychological safety, and system complexity. California Management Review, 45, 136-158.

Robertson, B. J. (2015). Holacracy: the revolutionary management system that abolishes hierarchy. London: Penguin.

Rüther, C. (2017). Soziokratie: Ein Organisationsmodell. Grundlagen, Methoden und Praxis. BoD - Books on Demand.

Schein, E.H., \& Bennis, W. G. (1965). Personal and organizational change through group methods: The laboratory approach. New York: Wiley.

Schepers, J., De Jong, A., Wetzels, M., \& De Ruyter, K. (2008). Psychological safety and social support in groupware adoption: a multi-level assessment in education. Computers \& Education, 51, 757-775.

Schermuly, C.C. (2016). New Work-Gute Arbeit gestalten: Psychologisches Empowerment von Mitarbeitern. Bd. 10167. Freiburg: Haufe-Lexware.

Scholl, W. (2020). New Team-Work - eine neue Balance von Gruppe und Führung. Organisationsberatung Supervision Coaching, 27(2), 139-154. https://doi.org/10.1007/s11613-020-00645-9.

Siemsen, E., Roth, A. V., Balasubramanian, S., \& Anand, G. (2009). The influence of psychological safety and confidence in knowledge on employee knowledge sharing. Manufacturing \& Service Operations Management, 11, 429-447.

Stalmeijer, R.E., Gijselaers, W.H., Wolfhagen, I., Harendza, S., \& Scherpbier, A. (2007). How interdisciplinary teams can create multi-disciplinary education: the interplay between team processes and educational quality. Medical Education, 41, 1059-1066.

Tajfel, H. (1982). Social psychology of intergroup relations. Annual review of psychology, 33(1), 1-39.

Tajfel, H. \& Turner, J. (2004). An integrative theory of intergroup conflict. In M. J. Hatch \& M. Schultz (Hrsg.) Organizational Identity, (S. 56-65). Oxford: Oxford University Press.

Tubre, T.C., \& Collins, J.M. (2000). Jackson and Schuler (1985) revisited: a meta-analysis of the relationships between role ambiguity, role conflict, and job performance. Journal of Management, 26, 155-169.

Turner, J.C. (1984). Psychological group formation'. The social dimension: European developments in social psychology. Bd. 2 (S. 518-538). Cambridge: Cambridge University Press.

Turner, A.N., \& Lawrence, P.R. (1965). Industrial jobs and the worker: an investigation of response to task attributes. Boston: Harvard University.

Tynan, R. (2005). The effects of threat sensitivity and face giving on dyadic psychological safety and upward communication. Journal of Applied Social Psychology, 35, 223-247.

Van Der Vegt, G. S., \& Bunderson, J. S. (2005). Learning and performance in multidisciplinary teams: the importance of collective team identification. Academy of management Journal, 48(3), 532-547.

Wageman, R. (1995). Interdependence and group effectiveness. Administrative Science Quarterly, 40(1), 145-180. https://doi.org/10.2307/2393703.

Walumbwa, F. O., \& Schaubroeck, J. (2009). Leader personality traits and employee voice behavior: mediating roles of ethical leadership and work group psychological safety. Journal of Applied Psychology, $94,1275-1286$.

Wang, J., \& Leung, K. (2011). The antecedents and consequents of individual psycho-logically safe communication climate. Paper presented at the Annual Conference of Academy of Management, San Antonio.

West, M. A. (1990). The social psychology of innovation in groups. In M. A. West \& J.L. Farr (Hrsg.), Innovation and creativity at work: psychological and organizational strategies (S. 309-333). Chichester: Wiley. 
West, M.A., \& Richter, A.W. (2008). Climates and cultures for innovation and creativity at work. In J. Zhou \& C.E. Shalley (Hrsg.), Handbook of organizational creativity (S. 211-236). New York: Taylor \& Francis.

Whitworth, E., \& Biddle, R. (2007). The social nature of agile teams. Agile, 2007, 26-36. IEEE.

Wong, A., Tjosvold, D., \& Lu, J. (2010). Leadership values and learning in China: the mediating role of psychological safety. Asia-Pacific Journal of Human Resources, 48, 86-107.

$\mathrm{Xu}$, Y., \& Yang, Y. (2010). Student learning in business simulation: an empirical investigation. Journal of Education for Business, 85, 223-228.

Yalom, I. D., \& Leszcz, M. (2020). The theory and practice of group psychotherapy. London: Hachette.

Zhang, Y.X., Fang, Y.L., Wie, K. K., \& Chen, H.P. (2010). Exploring the role of psychological safety in promoting the intention to continue sharing knowledge in virtual communities. International Journal of Information Management, 30, 425-436.
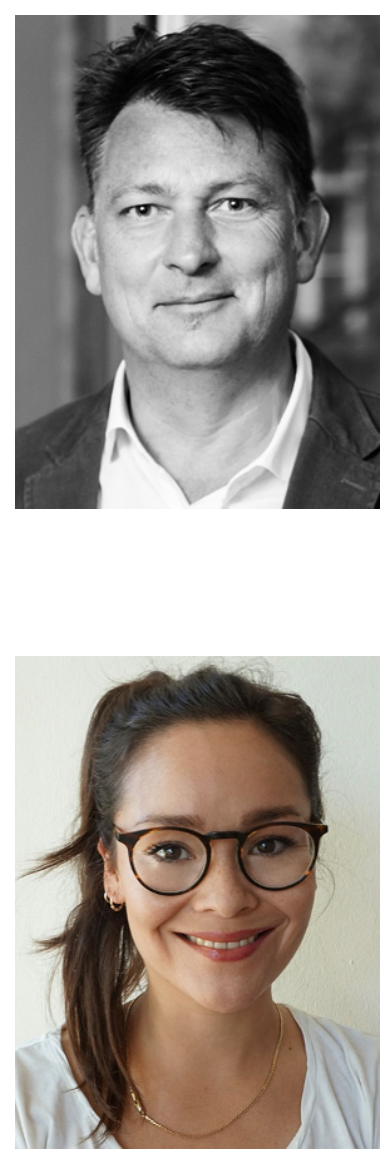

PD Dr. Thomas Bachmann Dipl.-Psych., Gründungsmitglied und Partner der artop GmbH; Studium der Arbeits- und Organisationspsychologie, Klinische Psychologie sowie Informatik, wissenschaftl. Mitarbeiter am Lehrstuhl für Methodenlehre und Statistik, 1997 Promotion in der Kognitiven Psychologie, 2019 Habilitation u. a. zu Systemtheorie und Gestalttherapie. Seit 1993 Berater, Trainer und Coach (DBVC, ICF), seit 2001 Ausbilder und Lehrtrainer für Coaches und Berater.

Katherina Quispe Bravo M.Sc. Wirtschaftspsychologin, Gründerin von Bravo Leadership; Seit 2019 Systemische Coach und Trainerin für Führung in dynamischen Organisationen. Seit 2020 Promovendin am Lehrstuhl Psychologie an der Humboldt-Universität zu Berlin. Seit 2021 Dozentin an der SRH Berlin University of Applied Sciences im Fach „Trust and Collaboration“. 